\title{
Evaluation of a New Phenotypic Method to Screen for OprD-Deficient Mutant Strains of Pseudomonas aeruginosa
}

\author{
Omnia M. Ahmed*, A.A Manal and A.G. Samia \\ Clinical Pathology Department, Faculty of Medicine, Ain shams University, Egypt \\ *Corresponding author
}

\begin{tabular}{|c|}
\hline Keywords \\
\hline $\begin{array}{l}\text { OprD-Deficient } \\
\text { Mutant, } \\
\text { Pseudomonas } \\
\text { aeruginosa } \\
\text { Carbapenemase }\end{array}$ \\
\hline Article Info \\
\hline $\begin{array}{l}\text { Accepted: } \\
20 \text { January } 2017 \\
\text { Available Online: } \\
10 \text { February } 2017\end{array}$ \\
\hline
\end{tabular}

\section{Keywords}

OprD-Deficient

Mutant,

Pseudomonas

aeruginosa

Article Info

Accepted:

20 January 2017

Online:

and

\section{A B S T R A C T}

Development of carbapenem resistance is common in Pseudomonas aeruginosa. This resistance, which is due mainly to alteration of the $\operatorname{OprD}$ porin, the specific uptake pathway of carbapenems, may also result from acquisition of foreign genes encoding Ambler class A, class B, or class D B-lactamases. These enzymes able to hydrolyze carbapenems at various degrees. Thus, detection of carbapenemase producers in clinical laboratories is of utmost importance for the determination of appropriate therapeutic schemes and the implementation of infection control measures. In order to improve the detection of carbapenemase producers, various inhibitor-based tests and enzymatic assays (e.g., NP-Carba) have been proposed as a first screening step prior to the use of confirmatory molecular techniques. However, because most of imipenem-non susceptible strains are just $O p r D$-deficient mutants, these phenotypic or enzymatic tests usually yield low rates of positivity.A new test is developed in order to screen for $O p r D$-deficient mutants thus discriminating carbapenemase producing $P$. Aeruginosa strains from nonproducers. This test combines imipenem and cloxacillin, a strong inhibitor of intrinsic cephalosporinase AmpC. It is based on the observation that imipenem resistance resulting from $O p r D$ deficiency requires constitutive and/or carbapenem-induced overproduction of AmpC. Therefore, inhibition of AmpC by cloxacillin is expected to restore partial or complete sensitivity to imipenem in $\mathrm{OprD}$-deficient strains but not in carbapenemase positive strains. The aim of this study was to evaluate the performance of a simple, inexpensive detection method applicable in medical laboratories that used combined disk testing of Imipenem and cloxacillin, in order to discriminate carbapenemase producing $P$. aeruginosastrains from non-producers $(\mathrm{OprD}$-deficient mutants).Fifty clinical isolates of imipenem resistant $P$. aeruginosa, which were well characterized by MHT for carbapenamse production and by real time PCR for $\left(\boldsymbol{b} \boldsymbol{l a}_{\mathrm{IMP}}\right)$ gene and $\left(\boldsymbol{b} \boldsymbol{a}_{\mathrm{KPC}}\right)$ gene from previous studies, were used. Combined test testing (CDT) using a carbapenem disk (imipenem) supplemented with various loads of cloxacillin was assessed. Out of 50 P.aeruginosa isolates, 15 (30\%)isolates were positive by Imipenem-Cloxacillin CDT at conc. 4000.This CDT detected $\mathrm{OprD}$-deficient mutants which were non carbapenamse producer strains. In comparison to (bla $\boldsymbol{a}_{\mathrm{IMP}}$ gene positivity and (bla $\left.\boldsymbol{a}_{\mathrm{KPC}}\right)$ gene positivity, and MHT results, sensitivity and specificity of imipenem-cloxacillin at conc. 4000ug were $93.33 \%$ and $75 \%$ respectively. In conclusion, the Imipenem-Cloxacillin at conc. 4000 test is a simple and inexpensive presumptive method that can be added to the standard antibiogram for routine screening of $O p r D$-deficient mutants thus discriminating carbapenemase producing $P$. aeruginosa strains from non-producers. 


\section{Introduction}

Pseudomonas aeruginosa ( $P$. aeruginosa) is an opportunistic pathogen associated with a range of nosocomial infections. This microorganism is noted for its intrinsic resistance to antibiotics and for its ability to acquire genes encoding resistance determinants. Among the beta-lactam antibiotics, carbapenems with anti pseudomonal activity are important agents for the therapy of infection due to $P$. aeruginosa (Meletis et al., 2012).

Development of carbapenem resistance is common in $P$. aeruginosa. This resistance, which is due mainly to alteration of the $O p r D$ porin, the specific uptake pathway of carbapenems in $P$. aeruginosa, may also result from acquisition of foreign genes encoding Ambler class A, class B, or class DBlactamases. These enzymes are able to hydrolyze carbapenems at various degrees (Cornaglia et al., 2011).

Over the last decade, strains producing metallo-beta-lactamases (MBLs) of different types (e.g., AIM, FIM, GIM, IMP, NDM, SPM, and VIM) have been reported with an increasing frequency in $P$. aeruginosa worldwide (Pollini et al., 2013). In order to improve the detection of carbapenemase producers, various inhibitor-based tests and enzymatic assays (e.g., NP-Carba) have been proposed as a first screening step prior to the use of confirmatory molecular techniques (Dortet et al., 2012).

However, because most of imipenem-non susceptible strains are just OprD-deficient mutants, these phenotypic or enzymatic tests usually yield low rates of positivity. Moreover, phenotypic assays cannot specify types within each class of carbapenemases, such as IMP, VIM, NDM, SIM, and GIM in MBLs. Therefore molecular confirmation of carbapenemases is recommended for suspected carbapenemase-producing strains. However, although molecular detection methods such as PCR and sequencing of carbapenemase genes are reliable for confirmation of carbapenemases, it is difficult to perform such tests in routine clinical microbiology laboratories because of the skill level required, the higher cost, and the special equipment required (Nordmann et al., 2012).

A new test is developed in order to discriminate carbapenemase producing strains from non-producers. This test combines imipenem and cloxacillin, a strong inhibitor of intrinsic cephalosporinase AmpC. It is based on the observation that imipenem resistance resulting from $O p r D$ deficiency requires constitutive and/or carbapeneminduced overproduction of AmpC. Therefore, inhibition of AmpC by cloxacillin is expected to restore partial or complete sensitivity to imipenem in $O p r D$-deficient strains but not in carbapenemase positive strains (Fournier et al., 2013).

The aim of this study is to evaluate the performance of a simple, inexpensive detection method applicable in medical laboratories that combines imipenem and cloxacillin, as a strong inhibitor of intrinsic cephalosporinase AmpC, in order to discriminate carbapenemase producing Pseudomonas aeruginosa strains from nonproducers (OprD-deficient mutants).

\section{Materials and Methods}

Fifty clinical isolates of imipenem non susceptible Pseudomonas aeruginosa strains that were well characterized from previous studies (Taher, 2016) that were conducted in Main Microbiology Laboratory, Ain Shams University Hospitals Cairo, Egypt, were used. Isolates were well characterized for production of carbapenemase by Modified Hodge test (MHT), MBL production by 
carbapenem / Dipiclonic acid(DPA) CDT with $\left(b l a_{\mathrm{IMP}}\right)$ gene confirmation and KPC production by carbapenem / Aminophenylboronic Acid (APB)CDT with $\left(b l a_{\mathrm{KPC}}\right)$ gene confirmation by real time PCR.

Results from previous studies (Taher, 2016) were used. Out of 50 P. aeruginosa isolates, $33(66.0 \%)$ isolates were positive (carbapenemase producers) by Modified Hodge test (MHT). Out of the 33 isolates, 22 isolates were positive for target genes $\left(b{ }^{\mathrm{IMP}}\right.$ gene, $b l a_{\mathrm{KPC}}$ genes) by real time PCR, and eleven isolates were negative for target genes in these study. Out of $50 \mathrm{P}$. aeruginosa isolates, $b a_{\mathrm{IMP}}$ gene was detected in 13 $(26.0 \%)$ isolates and all were positive for carbapenemase production by MHT. Out of these 13 isolates, ten isolates were positive by carbapenem / DPA acid CDT. Out of 50 P.aeruginosa isolates, bla $a_{\mathrm{KPC}}$ gene was detected in $15(30 \%)$ isolates different from that were positive for $b l a_{\mathrm{IMP}}$ gene by real time PCR, nine isolates of them only were positive by MHT. Out of these 15 isolates, eleven isolates were positive by carbapenem /APB acid CDT.

Isolates were stored at $-70 \circ \mathrm{C}$ for further processing.

\section{Combined disk test (CDT) Imipenem- Cloxacillin}

Combined test testing (CDT) using Imipenem supplemented with different loads of cloxacillin was assessed for the ability of screening for $O p r D$-deficient strains thus discriminating carbapenemase producing Pseudomonas aeruginosa strains from nonproducers. Then results of CDT were compared to results of real time PCR for detection of $\left(b l a_{\mathrm{IMP}}\right)$ gene and $\left(b l a_{\mathrm{KPC}}\right)$ gene as a reference method.A positive imipenemcloxacillin combined test testing (CDT) denotes non carbapenamase producing $P$. aeruginosa isolates (OprD-deficient mutants) (Fournier et al., 2013).

This test is based on the observation that imipenem resistance resulting from $\mathrm{OprD}$ deficiency requires constitutive and/or carbapenem-induced overproduction of $\mathrm{AmpC}$, therefore inhibition of $\mathrm{AmpC}$ by cloxacillin is expected to restore partial or complete sensitivity to imipenem in $O p r D-$ deficient strains but not in carbapenemase positive strains. A $0.5 \mathrm{McFarland}$ suspension from each test strain was inoculated on Muller Hinton agar plate according to the CLSI guideline. Five different disks were placed on the Muller Hinton agar plate as follows: a 10$\mu \mathrm{g}$ Imipenem (IPM) disk (Oxoid, UK), a 10$\mu \mathrm{g}$ Imipenem (IPM) disk supplemented with acloxacillin (cloxacillin sodium salt monohydrate) (Sigma-Aldrich, Germany) load of $100 \mathrm{mg} / \mathrm{ml}$ with an end concentration of 1,000 $\mu \mathrm{g}$ per disk, a 10- $\mu \mathrm{g}$ Imipenem (IPM) disk supplemented with acloxacillin load of $200 \mathrm{mg} / \mathrm{ml}$ with an end concentration of 2,000 $\mu \mathrm{g}$ per disk, a 10- $\mu \mathrm{g}$ Imipenem (IPM) disk supplemented with acloxacillin load of $300 \mathrm{mg} / \mathrm{ml}$ with an end concentration of 3,000 $\mu \mathrm{g}$ per disk and a $10-\mu \mathrm{g}$ Imipenem (IPM) disk supplemented with a cloxacillin load of $400 \mathrm{mg} / \mathrm{ml}$ with an end concentration of $4,000 \mu \mathrm{g}$ per disk. After 18 to $20 \mathrm{~h}$ of incubation at $37^{\circ} \mathrm{C}$, the difference between the zone diameters around imipenem disks alone and supplemented with cloxacillin at concentration of $1,000 \mu \mathrm{g}, 2,000 \mu \mathrm{g}, 3,000 \mu \mathrm{g}$ and $4,000 \mu \mathrm{g}$ per disk is measured in millimeters and expressed as $\chi_{\mathrm{d}}$ in millimeters. A cutoff value of $5 \mathrm{~mm}$ is considered. An increase in the zone size of $>$ $5 \mathrm{~mm}$ for imipenem in the presence of cloxacillin compared with that of the drug alone was considered a positive result = absence of carbapenemase activity and no or an increase in the zone size of $<5 \mathrm{~mm}$ for imipenem in the presence of cloxacillin compared with that of the drug alone was 
considered a negative result $=$ carbapenemase producer strain (Fournier et al., 2013).

Comparison of the results of ImipenemCloxacillin CDT at conc of 4000 with results of APB and DPA CDT from previous studies (Taher, 2016) using the algorithm from (Pasteron et al., 2011) (Figure.1) for discriminating of carbapenemase producers from non-producers strains was made.

\section{Statistics}

Categorical variables were expressed as number (\%).The diagnostic test evaluation; sensitivity, specificity and the positive and negative predictive values, accuracy and Cohen's Kappa coefficient (k) for agreement were calculated for determining the diagnostic validity of the test. All the analyses were performed with commercially available software (SPSS version 20, SPSS, Inc., Chicago, IL, USA).

To calculate sensitivity, specificity, negative predictive value, positive predictive value and accuracy, results of ( $\left.b l a_{\mathrm{IMP}}\right)$ gene and $\left(b l a_{\mathrm{KPC}}\right)$ gene positivity by real time PCR were summed to represent the true positive (28) isolates and the results of MHT positivity (33) was differentiated to 28 true positive and five isolates false positive.

\section{Results and Discussion}

\section{Phenotypic and molecular testing}

In this study, Out of the $50 P$. aeruginosa isolates nine $(18.0 \%)$ were positive by Imipenem-Cloxacillin CDT at concentration $1000 u g$, increased to be $11(22.0 \%)$ at concentration $2000 \mathrm{ug}$, also raised to be 14 $(28.0 \%)$ at concentration 3000ug, and increased to be $15(30.0 \%)$ at concentration 4000ug denoting OprD-deficient strains. All 15 positive isolates were negative by MHT and DPA CDT. Out of the 15 positive isolates for Imipenem-Cloxacillin CDT, 11 were negative for both genes (bla $a_{\mathrm{IMP}}$ gene and bla $_{\mathrm{KPC}}$ gene by real time PCR, the remaining four isolates contained bla $a_{\mathrm{KPC}}$ gene. Three isolates out of these four isolates were positive by APB CDT.

In the current study, two isolate were positive by both Imipenem-Cloxacillin and APB CDT and were negative for $b l a_{\mathrm{KPC}}$ gene.

In the current study, eleven isolates were negative by PCR for both genes and by Imipenem-Cloxacillin CDT but were positive by MHT.

On comparing sensitivity results of Imipenem-Cloxacillin combination-disk test at concentration of $1000 \mu \mathrm{g}, 2000 \mu \mathrm{g}, 3000$ $\mu \mathrm{g}$ and $4000 \mu \mathrm{g}$ respectively according to summation of results PCR bla-IMP gene and bla KPC gene and MHT results; the results were $(77.78 \%, 82.35 \%, 90.32 \%$ and $93.33 \%)$ respectively, specificity results were $(64.29 \%$, $68.75 \%, 73.68 \%$ and $75 \%$ ) respectively, negative predictive value results were (52.94\%, 64.71\%, $82.35 \%$ and $88.24 \%$ ) respectively, positive predictive value results were $(84.85 \%, 84.85 \%, 84.85 \%$ and $84.85 \%)$ respectively and the accuracy results were $(64 \%, 73 \%, 82 \%$ and $88 \%)$ respectively.

Carbapenems, among the beta-lactams, are the most effective against Gram-positive and Gram negative bacteria presenting a broad spectrum of antibacterial activity. Gramnegatives commonly recruit mechanisms to overcome the effect of carbapenem antibiotics. Certain species are able to prevent carbapenems reaching their PBPs by diminishing the permeability of their outer membrane. $O p r D$ for example, is an outer membrane porin of $P$. aeruginosa through which carbapenems enter its periplasmic space where PBPs are located. Consequently, 
the diminished expression or loss of this porin leads to carbapenem resistance without concurrent resistance to other beta-lactams. The most common mechanism of resistance to the carbapenems (including imipenem) in $P$. aeruginosa is loss or alteration of the outer membrane porin protein $O p r D$, the major portal for entry for carbapenems. While not providing the high-level resistance seen in MBL-producers, loss of $O p r D$ function is the major determinant of non-MBL-mediated resistance to these agents, often seen operating in conjunction with other mechanisms (e.g., ampC or MexAB-OprM). Indeed, carbapenem resistance resulting from loss of $O p r D$ requires the presence of AmpC (inducible or stably derepressed) (Poole, 2011).

So a new phenotypic test is needed to detect this porin loss in order to discriminate carbapenamse producer strains from nonproducers (Meletis, 2016).

The aim of this study was to evaluate a new phenotypic method toscreen for OprDdeficient mutants thus discriminating carbapenemase producing $P$. aeruginosa strains from non-producers.Fifty clinical isolates of imipenem non susceptible $P$. aeruginosa that are well characterized from previous studies (Taher, 2016) for carbapenemase production by MHT, MBL production by DPA with $\left(\right.$ bla $\left._{\mathrm{IMP}}\right)$ gene and KPC production by APB with (bla $\left.a_{\mathrm{KPC}}\right)$ gene by real time PCR were used. Combined test testing (CDT) using Imipenem supplemented with different loads of cloxacillin was assessed for the ability of detection of $O p r D$ deficient strains.

In this study, Out of the $50 P$. aeruginosa isolates $9(18.0 \%)$ were positive by Imipenem-Cloxacillin CDT at concentration $1000 \mathrm{ug}$, increased to be $11(22.0 \%)$ at concentration $2000 \mathrm{ug}$, also raised to be 14
(28.0\%) at concentration 3000ug, and increased to be $15(30.0 \%)$ at concentration 4000ug denoting OprD-deficient strains. All 15 positive isolates were negative by MHT and DPA CDT. In a study made by Fournier et al., (2013), they tested a panel of 118 imipenem-non susceptible clinical strains of $P$. aeruginosa characterized by the French National Reference Centre for Antibiotic Resistance. All the $\beta$-lactamase genes were characterized by PCR and DNA sequencing. Over expression of genes encoding AmpC and efflux systems was assessed by real-time quantitative PCR after reverse transcription. They evaluated the same CDT using imipenem and cloxacillin to discriminate between carbapenemase-producing $(n=56)$ and non-producing $(\mathrm{n}=62)$ strains of $P$. aeruginosa. They used Sequencing of the porin OprDgene as a reference method for Imipenem-Cloxacillin CDT. Sequencing of the porin $\operatorname{OprD}$ gene in 20 randomly selected strains belonging to non carbapenemase producers group showed that all were $O p r D$ deficient.

Cloxacillin as a strong inhibitor of intrinsic cephalosporinase AmpC, is widely investigated to study the association between AmpC over production and decreased susceptibility to Imipenem. Guti'errez et al., (2007) reported an association between carbapenem resistance and AmpC overproduction in $P$. aeruginosa (Guti'errez et al., 2007). Martínez et al., (2009b) made a study using cloxacillin-containing plates, the MICs of ceftazidime were decreased by at least two fold for $25(78 \%)$ out of 32 isolates, suggesting overproduction of AmpC that was confirmed by determination of B-lactamase activities. Twenty-one out of those 25 isolates over expressed the AmpC B-lactamase and had reduced MICs of ceftazidime but also of imipenem and cefepime after cloxacillin addition, suggesting the presence of an extended-spectrum AmpC (ESAC) beta- 
lactamases. In another study for Martínez et al., (2009a), they tested AmpC Betalactamase-specific in culture extracts by UV spectrophotometry after the incubation of culture extracts with 50 UM cloxacillin (AmpC Beta-lactamase inhibitor) for $15 \mathrm{~min}$. Extracts with $>90 \%$ reduction of Betalactamase activity after cloxacillin addition were considered to produce AmpC-type Blactamase as a major contributor of Blactamase activity.

Out of the 15 positive isolates for ImipenemCloxacillin CDT, 11 were negative for both genes $\left(\right.$ bla $_{\mathrm{IMP}}$ gene and $\left.b l a_{\mathrm{KPC}}\right)$ gene by real time PCR, the remaining four isolates contained $b l a_{\mathrm{KPC}}$ gene. Three isolates out of these four isolates were positive by APB CDT. This is in agreement with what Poole, 2011 stated in her review article; that the presence of KPC enzymes in carbapenemresistant isolates is often coupled with loss of the $O p r D$ outer membrane porin (Poole, 2011). In the current study, two isolate were positive by both Imipenem-Cloxacillin and
APB CDT and were negative for $b l a_{\mathrm{KPC}}$ gene. They were considered by the adopted algorithm as non carbapenamase producer, and confirmed by the negative results of both genes and MHT. In Pasteron et al., (2011) study, they found that Cephalosporinase overproducers were also positive in the APB test. They found that adding the results of cloxacillin CDT to APB testing reduced the number of false-positive results for KPC production by APB in the tested isolates.

In the current study, eleven isolates were negative by PCR for both genes and by Imipenem-CloxacillinCDT but were positive by MHT. These eleven isolates are considered carbapenamase producers but due to other genes less common than our target gene in this study. There are other genes coding for MBL-type (blaIMP, blaVIM, blaSPM, and blaAIM), and carbapenem-hydrolyzing class D (blaOXA-23, blaOXA-40, blaOXA-48, and blaOXA-58) Beta-lactamases (Martínez et al., 2009).

Fig.1 A Preliminary algorithm for screening ofcarbapenemase producing Pseudomonas aeruginosa isolates (Pasteron et al., 2011)

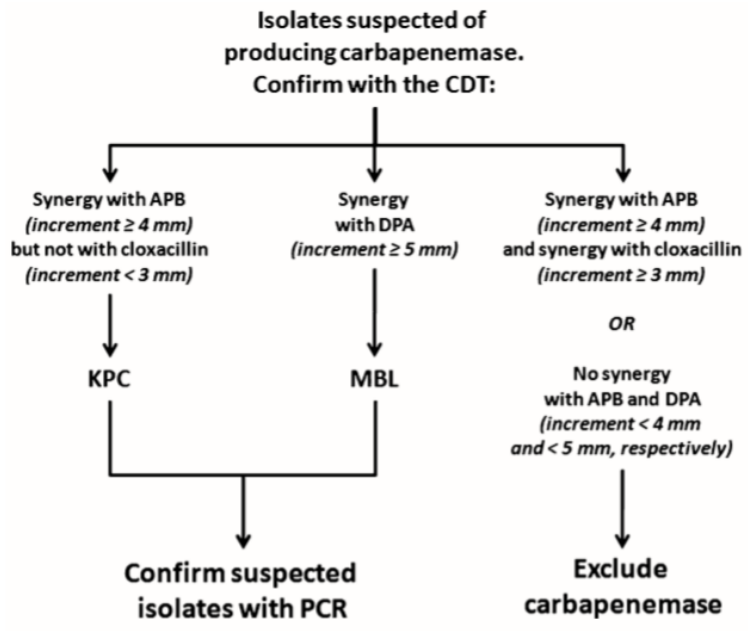

In the current study, the sensitivity and specificity of Imipenem-cloxacillin 4000
CDT is $93.3 \%$ and $75 \%$ respectively. Similarly, with a cloxacillin load of 
4,000ug/disk, Fournier et al., 2013 found that this very simple method showed a sensitivity and a specificity of $100 \%$, irrespective of the type of carbapenemase produced. In another study made by Pasteron et al., (2011), evaluated the ability of the combination disk test (CDT) meropenem-DPA, meropenemABP and meropenem-cloxacillin to discriminate between various carbapenemaseproducing $P$. aeruginosa isolates $[\mathrm{KPC}, \mathrm{n}=$ 36; metallo-b-lactamase (MBL), $\mathrm{n}=38$ ] and carbapenemase non-producers $(n=75)$. They reported the sensitivity and specificity of imipenem-cloxacillin at conc $3000 \mathrm{ug}$ to be 97\%. They concluded that thses CDTs could be very useful in daily practice to provide fast and reliable detection of KPC and MBL carbapenemases among $P$. aeruginosa isolates. Another study by Heinrichs et al., 2015, they evaluated the CDT using imipenem $(10 \mu \mathrm{g}) /$ cloxacillin $4,000 \mu \mathrm{g}$ and the Carba NP test to detect carbapenemaseproducing $P$. aeruginosa (CPPA). They made the tests on 188 imipenem non susceptible $P$. aeruginosa. CDT with cloxacillin showed a sensitivity and specificity of $97 \%$ and $96 \%$ compared to $88 \%$ and $99 \%$ for the Carba-NP test in order to detect CPPA. CDT with cloxacillin showed an NPV of $98 \%$, compared to $95 \%$ for the Carba NP test in order to detect CPPA. They concluded that Imipenem-cloxacillin test yielded good NPV to exclude the presence of MBL in imipenem non-susceptible isolates (Heinrichs et al., 2015). Inactivating mutations in OprD have been documented to confer resistance to imipenem and to a lesser extent to meropenem and doripenem. It is also remarkable that mutations leading to the upregulation of the MexAB-OprM active efflux system may increase the resistance to meropenem and doripenem but with no effect on the susceptibility of $P$. aeruginosa to imipenem, which is not a substrate for this system (Ocampo-Sosa et al., 2012).
In conclusion, the Imipenem-Cloxacillin combination disk test at conc4000 test is a simple and inexpensive presumptive method for routine screening of $o p r D$ deficient mutant P.aeruginosastrains. When CDT systematically added to the standard antibiogram by disk diffusion, the imipenemcloxacillin disk may allow the detection of potentially epidemic oprD deficient mutants with high specificity and sensitivity in the same day the drug susceptibility data are obtained. Thus, it may reveal useful to rapidly implement infection control measures and also to optimize the use of more sophisticated and expensive methods (e.g., PCR, chips) necessary to detect and characterize carbapenemase genes.

\section{References}

Cornaglia, G., Giamarellou, H., and Rossolini, G.M. 2011. Metallo- $\beta$-lactamases: a last frontier for $\beta$-lactams? Lancet Infect. Dis., 11(5): 381-93.

Dorlet, L., Poirel, L., and Nordmann, P. 2014. Worldwide dissemination of the NDM-type carbapenemases in Gramnegative bacteria. Biomed. Res. Int., 249856.

Fournier, D., Garnier, P., Jeannot, K., Mille, A., Gomez, A. and Plésiat, P. 2013. A Convenient Method To Screen for Carbapenemase-Producing Pseudomonas aeruginosa. J. Clin. Microbiol., 51(11): 3846.

Guti'errez, O., C. Juan, E. Cercenado, F. Navarro, E. Bouza, P. Coll, J.-L. Perez, and A. Oliver. 2007. Molecular epidemiology and mechanisms of carbapenem resistance in Pseudomonas aeruginosa isolates from Spanish hospitals. Antimicrob. Agents Chemother., 51: 4329-4335.

Heinrichs, A., Huang, T.D., Berhin, C., Bogaerts, P., and Glupczynski, Y. 2015. Evaluation of several phenotypic methods for the detection of carbapenemase-producing Pseudomonas aeruginosa. Eur. J. Clin. Microbiol. Infect. Dis., 34: 1467-1474.

Jesudason, M.V., Kandathi, A.J., and Balaji, V. 
2005. Comparison of the methods for the detection of the carbapenamase and the metallo-beta- lactamases production in the clinical isolates. Indian J. Med. Res., 121:780-3.

Martínez, J.R., Poirel, L., and Nordmann, P. 2009a.

Extended-Spectrum

Cephalosporinases in Pseudomonas aeruginosa Antimicrobial Agents and Chemotherapy, 53(5): 1766-1771.

Martínez, J.R., Poirel, L., and Nordmann, P. 2009b. Molecular Epidemiology and Mechanisms of Carbapenem Resistance in Pseudomonas aeruginosa. Antimicrobial Agents And Chemotherapy, 53(11): p. 4783-4788.

Meletis, G. 2016. Carbapenem resistance: overview of the problem and future perspectives. Ther. Adv. Infect. Dis., 3(1): 15-21.

Meletis, G., Exindari, M., Vavatsi, N., Sofianou, D. and Diza, E. 2012. Mechanisms responsible for the emergence of carbapenem resistance in Pseudomonas aeruginosa. Hippokratia, 16: 303-307.

Nordmann, P., Gniadkowski, M., Giske, G.C., Poirel, L., Woodford, N. and Miriagou, V. 2012. Identification and screening of carbapenemase-producing

Enterobacteriaceae. Clin. Microbiol. Infect., 18: 432-438.

Ocampo-Sosa, A., Cabot, G., Rodríguez, C., Roman, E., Tubau, F., Macia, M., Moya, B., Zamorano, L., Suárez, C., Peña, C., Domínguez, M., Moncalián, G., Oliver, A., Martínez-Martínez, L., and the Spanish Network for Research in Infectious Diseases (REIPI) 2012. Alterations of OprD in Carbapenem-Intermediate and -
Susceptible Strains of Pseudomonas aeruginosa Isolated from Patients with Bacteremia in a Spanish Multicenter Study. Antimicrobial Agents and Chemotherapy, 56(4): 1703-1713.

Pasteran, F., Veliz, O., Faccone, D., Guerriero, L., Rapoport, M., Mendez, T. and Corso, A. 2011. A simple test for the detection of KPC and metallo-b-lactamase carbapenemase producing Pseudomonas aeruginosa isolates with the use of meropenem disks supplemented with aminophenylboronic acid, dipicolinic acid and cloxacillin. Clin. Microbiol. Infect., (17)9: 1438-1441.

Pollini, S., Maradei, S., Pecile, P., Olivo, G., Luzzaro, F., Docquier, J., et al., 2013. FIM1 , a new acquired metallo- $\beta$-lactamase from a Pseudomonas aeruginosa clinical isolate from Italy. Antimicrob. Agents Chemother., 57: 410-416.

Poole, K. 2011. Pseudomonas aeruginosa resistance to the max. Frontiers In Microbiol., 2: 65.

Taher, A.M.O. 2016. Evaluation of Aminophenylboronic Acid for Detection of KPC Production in Pseudomonas aeruginosa. Int. J. Curr. Microbiol. App. Sci., 5(10): 1-9

Wolter, D.J., Khalaf, N., Robledo, I.E., Vazquez, G.J., Sante, M.I., Aquino, E.E., Goering, R.V., and Hanson, N.D. 2009. Surveillance of carbapenem resistant Pseudomonas aeruginosa isolates from Puerto Rican Medical Center Hospitals: dissemination of KPC and IMP-18 beta-lactamases. Antimicrob. Agents Chemother., 53: 16601664.

\section{How to cite this article:}

Omnia M. Ahmed, A.A Manal and Samia, A.G. 2017. Evaluation of a New Phenotypic Method to Screen for OprD-Deficient Mutant Strains of Pseudomonas aeruginosa. Int.J.Curr.Microbiol.App.Sci. 6(2): 1894-1901. doi: http://dx.doi.org/10.20546/ijcmas.2017.602.214 